\title{
Neurofibroma paciniano: relato de um caso raro de localização intra-oral
}

\section{Pacinian neurofibroma: report of a rare intraoral case}

\author{
Lélia B. Souza', Jamile M.B. Oliveira², \\ Tarsila M. C. Freitas ${ }^{3}$, Rejane A. Carvalho ${ }^{4}$
}

Palavras-chave: neurofibroma, corpúsculos de Pacini, mucosa oral.

Key words: neurofibroma, Pacinian corpuscle, oral mucosa.

\section{Resumo / Summary}

neurofibroma paciniano é uma lesão rara encontrada predominantemente em mãos, incluindo digitais, em nádegas e pés. As lesões podem ser solitárias ou múltiplas como componente da doença de Von Recklinghausen. Na cavidade oral, o neurofibroma paciniano é um achado incomum, existindo apenas um caso relatado na literatura mundial. 0 objetivo deste trabalho é apresentar um caso de neurofibroma paciniano localizado em mucosa gengival, bem como descrever as suas características clínicas e histopatológicas, contribuindo, assim, para um melhor conhecimento desta entidade.
Pacinian Neurofibroma is a rare lesion that has been reported predominantly on the hand, including the digits, on buttocks and feet. The lesions can be solitaries or multiples as part of the Von Recklinghausen's disease. The intraoral site is unusual and just one case has been related in the world's literature. The purpose of this report is to present one case of pacinian neurofibroma and to describe its clinical and histopathological features contributing for the best knowledge about this entity.

\footnotetext{
${ }_{1}$ Professora Doutora da Disciplina de Patologia O ral e do Programa de Pós-G raduação em Patologia O ral / UFRN.

${ }^{2}$ Aluna do Programa de Pós-graduação em Patologia O ral / UFRN (Mestrado).

${ }^{3}$ Aluna do Programa de Pós-graduação em Patologia Oral / UFRN (Mestrado).

${ }_{4}^{4}$ Professora Doutora do Programa de Pós-Graduação em Patologia Oral / UFRN. Instituição: Universidade Federal do Rio Grande do Norte.

Endereço para Correspondência: Drạ. Lélia Batista de Souza - Av. Salgado Filho, 1787 Lagoa Nova Natal RN 59056-000. Tel (0xx84) 215-4132/ 215-4138 - Fax: (0xx84) 215-4138

E-mail: leliasouza@dod.ufrn.br/ taifreitas@ig.com.br/ jamile.marinho@bol.com.br
}

Trabalho apresentado no III Congresso Brasileiro de Estomatologia/ XXI Jornada Brasileira de Estomatologia, Gramado-RS, 18 a 22 de Julho de 1995.

Artigo recebido em 20 de dezembro de 2002. Artigo aceito em 13 de fevereiro de 2003. 


\section{INTRODUÇÃO}

0 neurofibroma paciniano é um tumor benigno incomum que se origina a partir de terminações nervosas periféricas, constituindo uma variante histológica do neurofibroma por exibir componentes que se assemelham aos corpúsculos de Vater-Pacini. 0 objetivo deste trabalho é relatar um caso raro de neurofibroma paciniano de localização intra-oral, bem como descrever as suas características clínicas e histopatológicas, contribuindo para um maior conhecimento desta entidade.

\section{REVSÃO DA LITERATURA}

Os tumores neurais benignos são lesões oriundas dos nervos periféricos e representam cerca de $45 \%$ dos neoplasmas que atingem a região de cabeça e pescoço, sendo a alta incidência nesta área explicada pela quantidade relativamente grande de terminações nervosas periféricas agrupadas. Apesar de exibirem a mesma origem neural, apresentam notável heterogeneidade microscópica e patogenética que Ihes conferem um variado padrão de apresentação clínica e histológica. As lesões orais são raras. Acometem preferencialmente a língua ( $50 \%$ dos casos) e incluem o neurofibroma, shwannoma, o mixoma da bainha nervosa, o neuroma paliçado encapsulado, neuroma traumático, o tumor de células granulares e os neuromas mucosos da Síndrome de Neoplasias Endócrinas Múltiplas Tipo III ${ }^{1,2}$.

O neurofibroma é o tipo mais comum destes neoplasmas podendo ser solitários ou múltiplos como componente da neurofibromatose ${ }^{3}$. A pele é a localização mais freqüente, no entanto as lesões intra-orais não são incomuns, podendo ser periféricas, principalmente em língua, ou ainda centrais ${ }^{4}$. Do ponto de vista histológico, este tumor consiste de uma mistura de células de schwann, fibroblastos perineurais e endoneurais e, ocasionalmente, podem exibir estruturas que se assemelham a receptores pressóricos especializados denominados corpúsculos de Pacini, sendo, neste caso, conhecido como neurofibroma paciniano $0^{5,6}$.

O neurofibroma paciniano foi inicialmente descrito na literatura por Prichard e Custer em 1952, como lesões raras encontradas predominantemente em mãos, incluindo digitais, em nádegas e pés, acometendo preferencialmente adolescentes e adultos jovens. As lesões podem ser solitárias ou múltiplas como componente da doença de Von Recklinghausen caracterizada por neurofibromas múltiplos, manchas cutâneas café-com-leite, anormalidades ósseas, além de alterações do sistema nervoso central. Na cavidade oral, o neurofibroma paciniano é um achado incomum, podendo ser evidenciado em gengiva como uma massa tumoral exofítica, bem delimitada, de consistência mole e crescimento lento, envolvendo ocasionalmente o osso subjacente. 0 exame radiográfico revela uma imagem radiolúcida, podendo, ainda, ser observada divergência radicular ${ }^{2,6,7}$

0 diagnóstico diferencial desta entidade deve ser realizado com lesões como o granuloma piogênico, a lesão periférica de células gigantes e o fibroma ossificante periférico, lesões reacionais que acometem o tecido gengival e por exibirem aspectos clínicos semelhantes podem dificultar o seu diagnóstico ${ }^{4}$.

Com relação às características histopatológicas, 0 neurofibroma paciniano caracteriza-se por um padrão celular de arranjos lamelares concêntricos, compostos por células fusiformes, semelhantes aos corpúsculos de Vater-Pacini. Estes variam em tamanho e compreendem uma porção extensa dos elementos celulares, sendo circundados por um estroma denso com áreas hialinizadas. O s corpúsculos de Pacini são as maiores terminações neurais dos seres humanos, encontrando-se normalmente no tecido celular subcutâneo, em submucosas, subserosas de diversos órgãos, nas articulações e no mesentério em torno do pâncreas. Em algumas situações, como nestes casos de neurofibromas, formações semelhantes a tais corpúsculos podem ser evidenciadas ${ }^{2,8,9}$.

\section{APRESENTAÇÃO DO CASO CLÍNICO}

Paciente R.S., sexo feminino, 14 anos de idade, leucoderma, compareceu à Clínica de Estomatologia do Programa de Pós-Graduação em Patologia Oral da Faculdade de O dontologia da UFRN queixando-se de um "crescimento na gengiva". 0 exame clínico intra-oral revelou a presença de uma lesão exofítica localizada na gengiva vestibular, adjacente aos elementos 41 e 42, exibindo aspecto hiperplásico, coloração idêntica à mucosa circunjacente, medindo cerca de $1,5 \mathrm{~cm}$ de diâmetro, implantação séssil e consistência firme à palpação (Foto 1). Foi realizado exame radiográfico através de tomadas periapicais e Pôstero-Anterior de face não sendo detectadas alterações. Segundo relato da paciente a lesão era indolor, e apresentava uma evolução de aproximadamente três anos não estando associada a fatores traumáticos. Com a hipótese diagnóstica de fibroma ossificante periférico procedeu-se a realização de biópsia excisional.

O material recebido para exame anatomo-patológico exibia características macroscópicas de uma lesão de tecido mole, coloração acastanhada, consistência firme, superfície lobulada medindo $1,5 \times 1,3 \mathrm{~cm}$ em seus maiores diâmetros. À microscopia, observou-se uma neoplasia benigna de origem mesenquimal caracterizada pela proliferação de células fusiformes com núcleos alongados e citoplasma claro, arranjadas ora em estruturas pacinianas ora em lençóis. Em algumas áreas da lesão evidenciou-se disso ciação das fibras musculares pelas células neoplásicas. Completando o quadro histológico, observou-se a presença de tecido epitelial 
pavimentoso estratificado típico de revestimento da mucosa oral, bem como, fibras musculares dispostas na profundidade do espécime (Fotos 2 e 3). Frente a este quadro histopatológico, foi emitido o diagnóstico de Neurofibroma Paciniano. Mesmo tendo sido possível a identificação da lesão apenas pela coloração de rotina com hematoxilina-eosina, 0 espécime foi submetido à técnica imuno-histoquímica para a proteína S-100, apresentado, o tumor, reatividade positiva para este marcador de células neurais (Foto 4).

\section{DISCUSSÃO}

0 neurofibroma é um tumor benigno que se origina da bainha dos nervos periféricos, podendo ocorrer como parte da neurofibromatose tipo 1 (doença de Von Recklinghausen) ou, mais comumente como lesões únicas ${ }^{10}$.
Lesões solitárias ocorrem em muitas localizações anatômicas, incluindo a mucosa oral. Um amplo espectro de padrões histológicos e muitas variantes distintas têm sido descritos na literatura, incluindo a presença incomum dos corpúsculos de Vater Pacini, sendo, neste caso, conhecido como neurofibroma paciniano ${ }^{2,11,12}$.

O neurofibroma paciniano é uma lesão rara com ampla distribuição anatômica encontrada predominantemente em mãos, incluindo digitais, em nádegas e pés, estando em algumas situações associada à Neurofibromatose tipo 1. A localização intra-oral é incomum, sendo relatado apenas um caso na literatura envolvendo o osso maxilar². Tal raridade pode estar associada a dificuldade de diagnóstico e reconhecimento das estruturas de Vater Pacini, já que estes são preferencialmente observados no tecido celular subcutâneo, submucosas, subserosas, nas articulações e no mesentério.

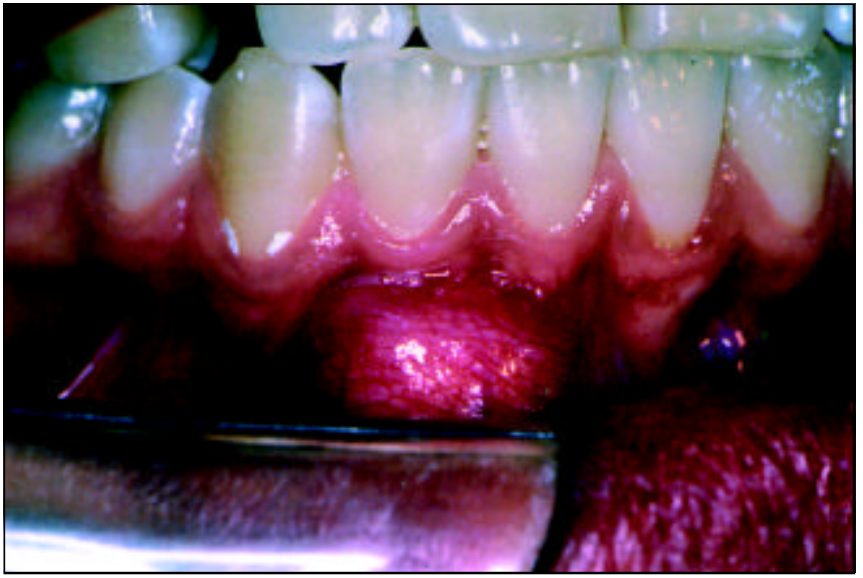

Foto 1. Aspecto clínico da lesão de neurofibroma paciniano exibindo superfície lisa, localizada na gengiva inserida.

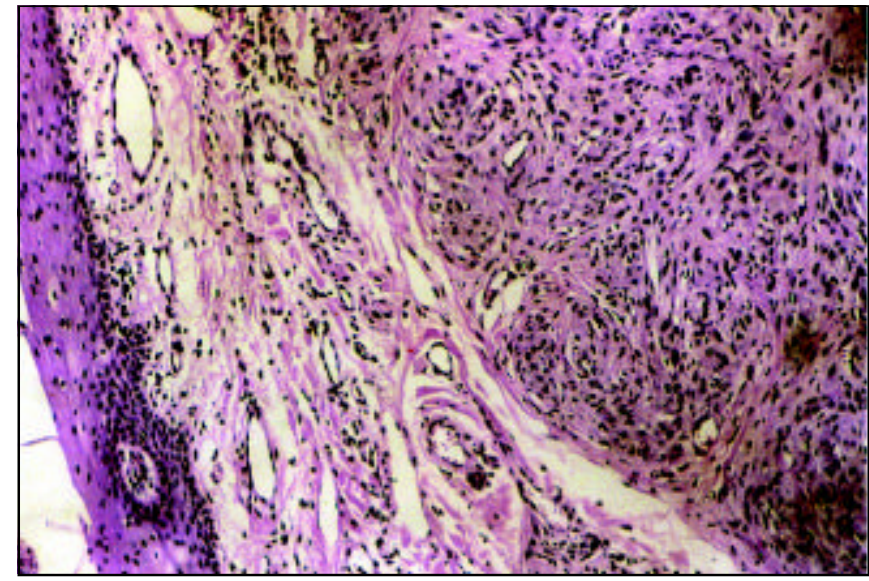

Foto 2. Aspecto histológico do neurofibroma paciniano evidenciandose a proliferação de células em arranjos lamelares concêntricos (H/E $100 x)$.

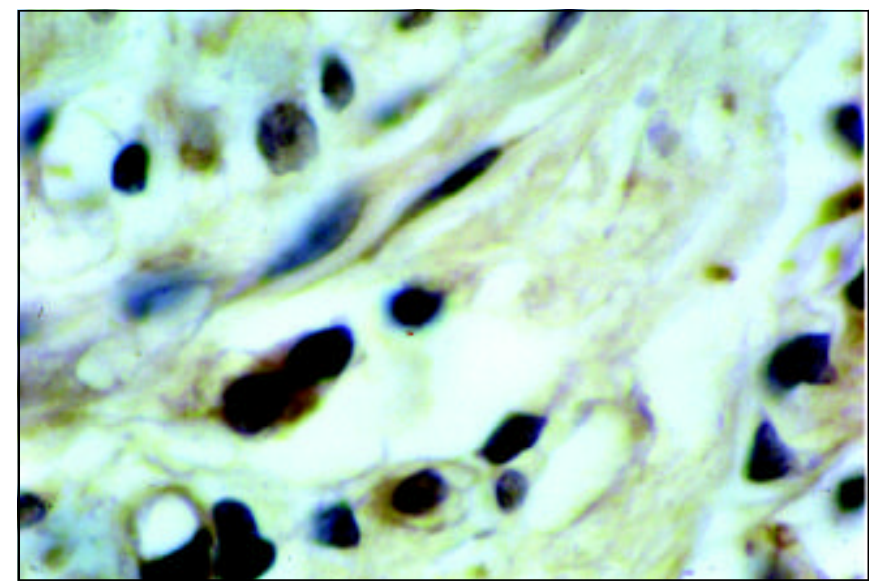

Foto 4. Expressão imuno-histoquímica para a proteína S-100 em neurofibroma paciniano (Estreptoavidina-biotina, 200x).
Foto 3. Fotomicrografia em maior aumento evidenciando-se estruturas

semelhantes aos corpúsculos de Vater-Pacini (H/E 200x).

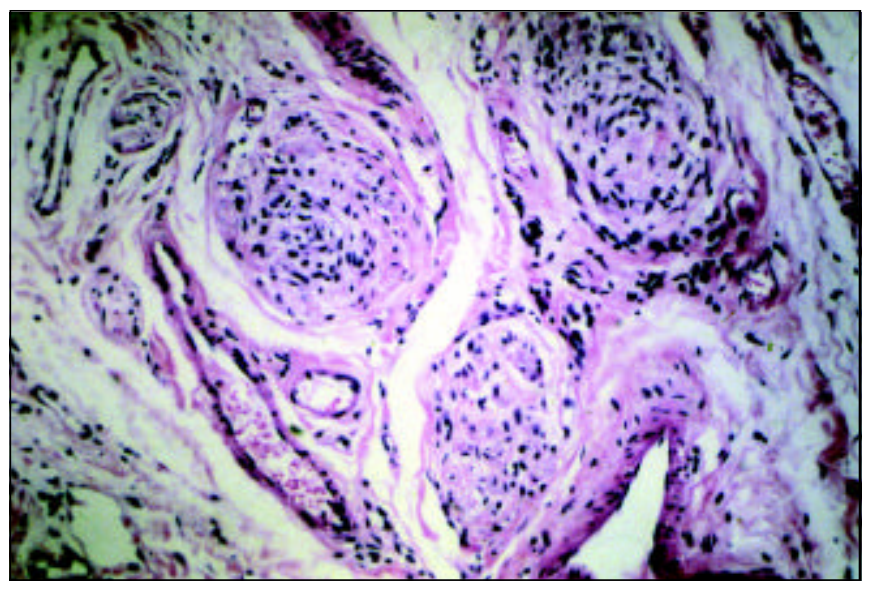


O caso ora relatado apresentava-se como uma lesão única de crescimento exofítico, localizado na gengiva vestibular adjacente aos elementos 41 e 42, sem evidência de neurofibromatose tipo 1 associada (Doença de Von Recklinghausen), corroborando com os achados de McCormack et al. ${ }^{7}$ que numa metanálise de 18 casos de neurofibroma paciniano não encontraram esta associação. Ao exame radiográfico não foi evidenciado comprometimento do osso subjacente indo de encontro aos achados de Toth, Long, Pleasants ${ }^{2}$, que evidenciaram imagem radiográfica radiolúcida ampla associada à lesão.

0 aspecto hiperplásico da lesão dificultou seu diagnóstico clínico, já que várias entidades que acometem a mucosa gengival exibem padrões semelhantes, o que nos levou às hipóteses de fibroma ossificante periférico e hiperplasia fibrosa. A tumefação local presente, por outro lado, conduziu-nos à suspeita de osteoma. Dessa forma, torna-se relevante a consideração destas lesões no estabelecimento do diagnóstico diferencial. 0 tratamento instituído para o neurofibroma paciniano, como preconizado pela literatura ${ }^{2,13}$, baseou-se na incisão cirúrgica local, através de uma biópsia excisional, sendo o espécime enviado para análise microscópica.

Os achados histopatológicos foram de fundamental importância para caracterização definitiva da lesão, corroborando com os achados de Bennin, Barsky, Salgiaz; McCormack et al. 7; Toth, Long, Pleasants's; Wolfgang Jurecka ${ }^{9}$ que evidenciaram a presença de um padrão celular de arranjos lamelares concêntricos, compostos por células fusiformes, semelhantes aos corpúsculos de Vater-Pacini.

Além do exame histopatológico, a marcação imunohistoquímica tem sido utilizada como recurso de diagnóstico na identificação de tumores neurais cutâneos e na determinação da linhagem celular. Os marcadores antigênicos capazes de determinar a origem neural incluem a proteína S-100, CD-57 e antígeno de membrana epitelial ${ }^{1,14}$. Neste caso, mesmo tendo sido possível a identificação da lesão apenas pela coloração de rotina com hematoxilinaeosina, o espécime foi submetido à técnica imunohistoquímica para a proteína S-100, apresentado o tumor reatividade positiva para este marcador de células neurais. A eficácia desta proteína, já apontada na literatura, foi testada por Chrysomali et al. ${ }^{1}$ em seu estudo de 77 casos de tumores neurais, sendo comprovada a sua capacidade na identificação de células de schwann em tumores mesenquimais de origem e diferenciação neural.

A ocorrência de uma grande porcentagem de tumores neurais, em região de cabeça e pescoço (45\%), é atribuída ao número relativamente grande de nervos periféricos agrupados nesta área ${ }^{2}$. Em nossa o pinião, a lesão relatada pode ter se originado de terminações nervosas oriundas do nervo alveolar inferior por um estímulo desconhecido, já que a paciente não relatou história de trauma durante a anamnese.

\section{COMENTÁRIOSFINAIS}

$\mathrm{Na}$ literatura existem poucos relatos a respeito do neurofibroma paciniano, sendo o mesmo reportado principalmente em mãos, pés e nádegas e mais raramente na cavidade oral. A lesão pode, em algumas situações, estar associada à Doença de Von Recklinghausen que caracterizase por lesões cutâneas múltiplas, o que torna relevante 0 minucioso exame clínico e físico dos pacientes. Não devemos esquecer, portanto, a importância do exame histopatológico que foi fundamental para o estabelecimento do diagnóstico definitivo pela presença das estruturas semelhantes aos corpúsculos de Vater-Pacini.

\section{REFERÊNCIASBIBUOGRÁFICAS}

1. Chrysomali E et al. Benign neural tumors of the oral cavity: a comparative immunohistochemical study. Oral Surg Oral Med Oral Pathol Oral Radiol Endod 1997; 84: 381-90.

2. Toth BB, Long WH, Pleasants JE. Central pacinian neurofibroma of the maxilla. Oral Surg Oral Med Oral Pathol Oral Radiol Endod 1975; 39:630-4.

3. Silva FM et al. Neurofibromatose múltipla. UNIMEP 2001; 13:14-16.

4. Neville BW et al. Tumores de tecidos moles. In: Patologia Oral e Maxilofacial. 2a ed. Rio de Janeiro, Guanabara: Koogan; 1998. p. 353-404.

5. Enzinger FM, Weiss SW. Soft tissues tumors. $2^{\underline{a}}$ ed. St. Louis: C.V. Mosby; 1988:1-741.

6. Regezi AJ, Sciubba JJ. Lesões do tecido conjuntivo. In: Patologia bucal: Correlações clínicopatológicas. 3a ed. Rio de Janeiro, Guanabara: Koogan; 2000:158-94.

7. McCormack K et al. Multiple hairy pacinian neurofibromas (nervesheat myxomas). J Am Acad Dermatol 1988; 18: 416-9.

8. Guidugli Neto J. Neurofibroma Paciniano (corpúsculos de Pacini alargados) intraperitoneal. Rev Paul Med 1990; 108:192-3.

9. Wolfgang Jurecka MD. Tactile corpuscle-like structures in peripheral nerve sheat tumors in plastic embedded material. Am J Dermatopathol 1988; 10:74-9.

10. Araújo ACPF et al. Neurofibromatose e gravidez: relato de caso. $R$ Saúde 1998; 12:47-51.

11. Bennin B, Barsky S, Salgia K. Pacinian Neurofibroma. Arch Dermatol 1976; 112:1557-8.

12. Shimoyama $T$ et al. Solitary neurofibroma of oral mucosa: a previously undescribed variant of neurofibroma. J Oral Science 2002; 44:59-63.

13. Campora E, Radici M, Campora L. Neurogenic tumors of the head and neck in children. Int J of Pediatric Otorhinolaryngol 1999; 49:231-3

14. Jordan RCK et al. Advanced diagnostic methods in oral and maxillofacial pathology. Part II: Immuohistochemical and immuofluorescent methods. Oral Surg Oral Med Oral Pathol Oral Radiol Endod 2002; 93:56-74. 